\title{
Remote Virtual Spinal Evaluation in the Era of COVID-19
}

\author{
JANG W. YOON, MD, MS, ${ }^{1}$ RACHEL L. WELCH, ${ }^{2}$ TODD ALAMIN, MD, ${ }^{3}$ WILLIAM F. LAVELLE, MD, ${ }^{4}$ \\ IVAN CHENG, MD, ${ }^{3}$ MICK PEREZ-CRUET, MD, MS, ${ }^{5}$ LOUIS C. FIELDING, MS, ${ }^{6}$ RICK C. SASSO, MD, ${ }^{7}$ \\ R.J. LINOVITZ, MD, ${ }^{8}$ KEE D. KIM, MD, ${ }^{9}$ WILLIAM C. WELCH, MD ${ }^{1}$ \\ ${ }^{1}$ Department of Neurosurgery, Perelman School of Medicine, University of Pennsylvania, Philadelphia, Pennsylvania, ${ }^{2}$ Yale College, Yale University, New Haven, \\ Connecticut, ${ }^{3}$ Department of Orthopedic Surgery and Neurosurgery, Stanford University Medical Center, Redwood City, California, ${ }^{4}$ Department of Orthopedic \\ Surgery, SUNY Upstate Medical University, Syracuse, New York, ${ }^{5}$ Department of Neurological Surgery, Oakland University William Beaumont School of \\ Medicine, Royal Oak, Michigan, ${ }^{6}$ Empirical Spine Inc., San Carlos, California, ${ }^{7}$ Indiana Spine Group, Carmel, Indiana, ${ }^{8}$ PJ Surgical Inc., Rancho Santa Fe, \\ California, ${ }^{9}$ University of California, Davis School of Medicine, Sacramento, California
}

\begin{abstract}
Background: With the COVID-19 pandemic disrupting many facets of our society, physicians and patients have begun using telemedicine as a platform for the delivery of health care. One of the challenges in implementing telemedicine for the spine care provider is completing a comprehensive spinal examination. Currently, there is no standardized methodology to complete a full spinal examination through telemedicine.

Methods: We propose a novel, remote spinal examination methodology that is easily implemented through telemedicine, where the patient is an active participant in the successful completion of his or her examination. This type of examination has been validated in a neurology setting. To facilitate the telemedicine visit, we propose that video instruction be shared with the patient prior to the telemedicine visit to increase the efficacy of the examination.

Results: Since the issuance of stay-at-home order across the states, many spine practices around the country have rapidly adopted and increased their telemedicine program to continue provide care for patients during COVID-19 pandemic. At a tertiary academic center in a busy metropolitan area, nearly 700 telemedicine visits were successfully conducted during a 4-week period. There were no remote visits being done prior to the shutdown.

Conclusions: Implementation of our proposed remote spinal examination has the potential to serve as a guideline for the spine care provider to efficiently assess patients with spine disease using telemedicine. Because these are only suggestions, providers should tailor examination to each individual patient's needs.

Level of Evidence: V.

Clinical Relevance: It is likely that physicians will incorporate telemedicine into health care delivery services even after the COVID-19 pandemic subsides because of telemedicine's efficiency in meeting patient needs. Using the standard maneuvers provided in our study, spine care providers can perform a nearly comprehensive spine examination through telemedicine. Further studies will be needed to validate the reproducibility and reliability of our methodology.
\end{abstract}

Other \& Special Categories

Keywords: telemedicine, spine examination, neurologic examination, motor examination, neurosurgery, spine surgery, virtual spine evaluation, virtual clinic, orthopedics

\section{INTRODUCTION}

The COVID-19 pandemic has swept through the world, and its full impact has yet to be realized. ${ }^{1}$ Although its immediate and short-term effects on the field of medicine have been tremendously disruptive, one potential benefit has been the increased use of telemedicine for outpatient clinical visits. $^{2-4}$ Using a platform that allows for the realtime synchronous transmission of video and audio information, spine surgeons are able to communicate with their patients and effectively collect pertinent history. ${ }^{5}$ There is not yet a standardized and established protocol to perform a comprehensive spinal evaluation using telemedicine alone. We have developed a novel protocol for remote spinal examinations for use in clinical practice through which spine care providers can perform consistent and reliable remote examinations for office-based exams.

\section{TELEMEDICINE PORTAL}

There are multiple telemedicine portals available for conducting HIPAA-compliant telemedicine spine visits, including but not limited to Zoom (San Jose, California), BlueJeans (San Jose, California), Teams (Redmond, Washington), Doxy.me (Rochester, New York), and EPIC (Verona, Wisconsin). Here, we describe the typical work flow of 


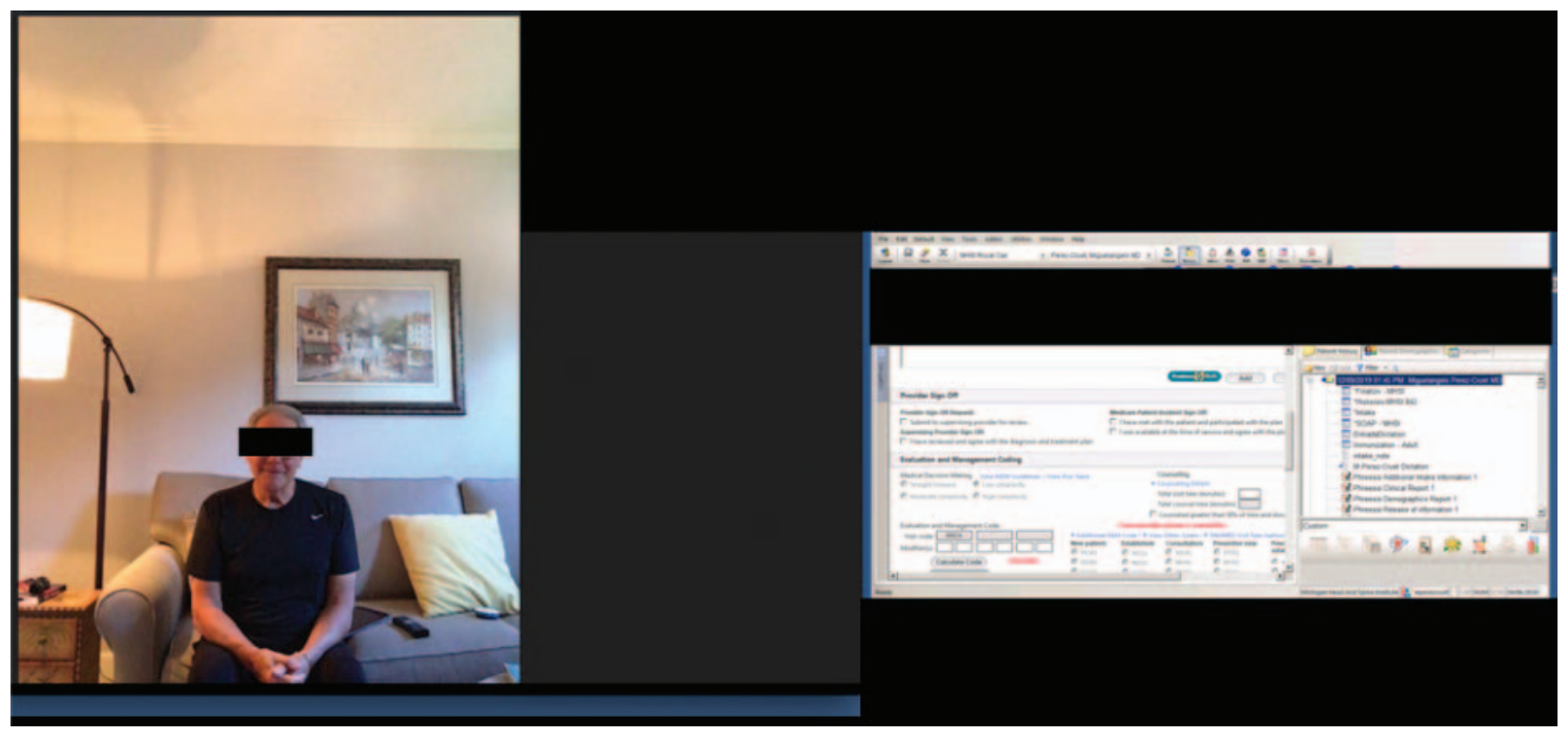

Figure 1. An example of a telemedicine clinic visit with a patient. The patient's EMR record can be reviewed by physician on the same computer screen.

using these portals to conduct spine clinics virtually. Prior to the initiation of the clinic, the physician and patient will need to download the relevant application onto their computers or smart phones. After scheduling the telemedicine clinic visit, the physician and patient are given a passcode to access the portal, and they are then connected via the telemedicine portal (Figure 1). Patients are asked to send their $\mathrm{CD}$ with their imaging studies to the office prior to the clinic visit, or to electronically load the results through their patient portal. Physicians can review the radiographic studies such as magnetic resonance, computed tomography, and $\mathrm{x}$-ray images, prior to or concurrent with the telemedicine examination.

The electronic medical record (EMR) is populated prior to the clinic visit by the patient with an intake form that shows all of that patient's medical history, including medications, allergies, past medical and surgical history, family medical history, and social history. The EMR is also populated with imaging results and neurophysiologic studies.

At the opening of the visit, it is incumbent on the provider or his or her staff to ask the patient for identification, and it is also good practice for the provider to wear an ID badge that is visible to confirm their identity. It is also important to discuss the inherent limitations of a video visit with the patient.

Once the patient is connected via the portal, a screen sharing option is available in some telemedicine portals that allows the physician to review the images with the patient. The physician can point out pertinent pathology to the patient via his computer cursor through the screen sharing function (Figure 2). Orders such as physical therapy, further imaging studies, pain management, and medications can be entered via the EMR portal. Medications are sent directly to the pharmacy of choice entered by the patient for pickup.

Following this protocol, one computer can be used to perform the telemedicine clinic visit, and other providers, such as residents, fellows, or advanced practice providers, can join the meeting in real time if they have been invited into the meeting. Coding for the visit can be performed via the EMR system, with entering the modifier GT to indicate that the visit was done via telemedicine. This system is very useful for following up on postoperative patients who do not require staple or suture removal. Additionally, this system has been useful in evaluating patients who have difficulty traveling to the office due to medical or logistical expense, inconvenience, or exposure during the current COVID-19 pandemic. In this manner, many patients have been effectively evaluated, treated nonoperatively, and/or scheduled for surgery.

Prior to the COVID-19 shutdown, approximately 1500 patients per month (750 new patient visits and 750 return visits) were seen at the Department of Neurosurgery at the University of Pennsylvania outpatient clinics. Since the onset of pandemic and shutdown order, in-person clinic visits have only been done on an emergency basis, which comprised 


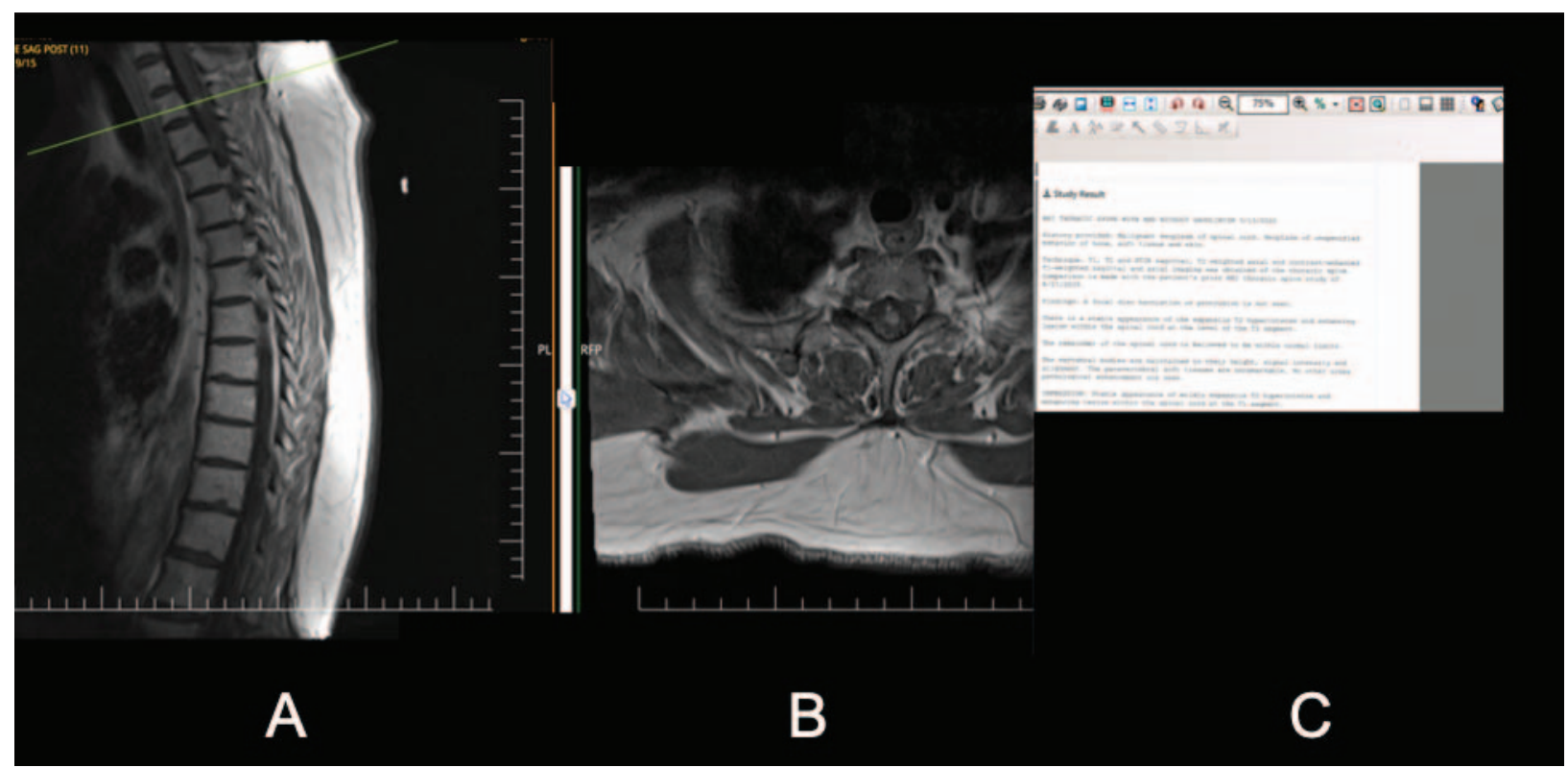

Figure 2. (A) Sagittal and (B) axial contrast-enhanced magnetic resonance image, and (C) radiographic report reviewed with a patient via shared screen option of telemedicine portal showing a lesion at the T1 level of spine.

fewer than 50 visits. However, with the rapid adoption and expansion of our telemedicine program, 695 telemedicine visits were completed during a period of 4 weeks from the middle of March to the beginning of April of 2020, enabling us to continue delivering spine care to patients remotely throughout the pandemic.

\section{RECOMMENDATIONS FOR REMOTE EXAMINATIONS}

Remote spinal examinations should be conducted via videoconference using a platform that conforms to each individual institution's policies for patient communication. Even though non-HIPAA-compliant platforms may be temporarily acceptable, these platforms will be expected to adhere to HIPAA compliance in the long term. ${ }^{6}$

It is imperative that a detailed history be obtained to help guide and augment the telemedicine examination. It is recommended that physicians provide instructions to the patient in advance, including videos and diagrams, so that the patient can efficiently perform the exercises during their telemedicine visit (see Supplemental Methods). Because the maneuvers are performed in a choreographed sequence, these videos promote the efficiency of the exam. Note that unless considered standard of care by an institution, Institutional Review Board approval may be required when providing these videos to patients. Furthermore, the exam itself is facilitated by having an assistant available, such as a family member, ${ }^{7}$ and physicians are encouraged during the exam to share their screen with patients to show them their pertinent radiographic studies. The examination can be more carefully guided by the patients' complaints reviewed below, including but not limited to dermatomal distribution of pain, dysesthesias, specific muscular weakness, and gait difficulties.

\section{GENERAL EXAMINATION}

The patient is asked to stand upright, and posture is assessed in anteroposterior and lateral planes. Cervical and lumbar movements and range of motion can also be assessed and recorded. Ambulation status can be assessed with the patient performing a natural walk and a "heel-toe" tandem gait.

\section{GAIT EXAMINATION}

The patient is asked to walk normally, and gait is examined for abnormalities, including wide or narrow base, spasticity, shuffling, steppage, waddling, and magnetic traits, in addition to sagittal and/or coronal plane deformity and antalgia. ${ }^{8}$

\section{SENSORY EXAMINATION}

A dermatomal pain diagram may be included for reference as well as a video demonstrating the examination technique. As applicable, the patient 
should complete any self-assessments, such as marking problematic areas. During the exam, the physician should ask the patient or assistant to lightly touch each dermatome on the patient with a pin or end of a paper clip; one extremity should be compared to the other side for reference.

\section{UPPER EXTREMITY SENSORY EXAMINATION}

- Specific areas that the patient may be asked to examine with a safety pin or open paper clip for each dermatome include the following:

- C2, C3: Back of the head

- C4: Trapezius

- C5: Shoulder

- C6: Biceps

- C7: Triceps

- C8: Ulnar side of hand

- T1: Middle of forearm (flexor side)

\section{LOWER EXTREMITY SENSORY EXAMINATION}

- Specific areas that the patient may be asked to examine for each dermatome include the following::

- L1: Just above iliac crest

- L2: Upper anterior thigh

- L3: Lower anterior thigh

- L4: Anterior/lateral aspect of thigh/shin/ medial lower leg

- L5: Lateral calf/top of foot/dorsal space between first and second toes

- S1: Lateral aspect of foot/bottom of foot/ calf

\section{MOTOR EXAMINATION}

Prior to motor testing, we suggest counseling the patients with regard to safety while performing maneuvers in the video and emphasizing that not all movements need to be attempted if too difficult given their functional status. For example, examinations should be tailored to each patient's ambulatory ability and need for assistive devices, because walking ability may inform selection and safety of the tests. In addition to the specific maneuvers described below, gait observation may additionally be indicative of motor deficits if an abnormal gait is apparent (e.g., gastrocnemius/soleus weakness, footdrop, or Trendelenburg gait). Direct video exami- nation using a commercial platform may also reveal muscle atrophy.

\section{UPPER EXTREMITY MOTOR EXAMINATION}

\section{Deltoid}

- For deltoid strength, ask a patient to pick up a known weight, such as a standard-sized can of soup (14.5-15 oz.), with his or her elbow flexed to $90^{\circ}$ and abducting the shoulder from zero to $90^{\circ}$ and then above the head. Alternatively, a greater weight may be used if clinically appropriate to a stronger patient so that sideto-side differences can be detected.

- Suggested scores are as follows:

- 5: Able to abduct the arm to beyond $90^{\circ}$

- 4: Unable to do the above task with a weight but able to do it without

- 3: Can raise the arm to $90^{\circ}$ but unable to raise beyond

- 2: Able to raise $<90^{\circ}$

- 1: Flicker of muscle activation

- 0: No movement

\section{Biceps}

- For biceps strength, ask a patient to flex his or her biceps at the elbow while holding a known weight, such as a can of soup. If the patient is unable to flex the arm at the elbow against gravity, then ask the patient to lay the arm on the table then flex the arm at the elbow.

- Suggested scores are as follows:

- 5: Able to flex the arm fully at the elbow with an object in his or her hand

- 4: Unable to do the above task with an object but able to do it without

- 3: Able to overcome gravity and flex the arm at the elbow, but quickly falls on the table.

- 2: Can only flex the arm at the elbow with gravity eliminated.

- 1: Flicker of muscle activation

- 0: No movement

\section{Triceps}

- For triceps strength, ask a patient to stand 2 to 3 feet away from a wall and push off of the wall (standing push-up). If he or she is unable to do this maneuver, then ask the patient to lie flat on his or her back and extend the arm fully into the air holding a known weight, such as a can of soup. 
- Suggested scores are as follows:

- 5: If able to push off his or her own body weight

- 4: Able to push the arm fully into the air from a supine position with a full range of motion holding a weight

- 3: Able to push the arm fully into the air from a supine position with a full range of motion without a weight

- 2: Only able to partially extend the arm into the air in a supine position

- 1: Flicker of muscle activation

- 0: No movement

\section{Wrist Flexors}

- Ask a patient to rest his or her forearm on a flat surface, such as a table, in a supinated position with the hand off the end of the table. While holding a known weight, have the patient flex the wrist. If the patient cannot flex the wrist against gravity, then the hypothenar side of the hand can be placed on the table, and wrist flexion can be performed parallel to the table surface.

- Suggested scores are as follows:

- 5: If able to do above maneuver with a weight and hold it for 5 seconds or longer

- 4: If able to do above maneuver with an object and hold it for 5 seconds or less

- 3: Cannot flex the wrist against gravity with an object in hand but can without it; has a full range of motion

- 2: Can only flex the wrist

- 1: Flicker of muscle activation

- 0: No movement

\section{Wrist Extensors}

- Ask a patient to rest the flexor side of the forearm on a flat surface, such as a table, with the hand off the end of the table, then extend the wrist while holding a known weight.

- Suggested scores are as follows:

- 5: Able to hold a weight fully extended for longer than 5 seconds

- 4: Able to hold a weight for less than 5 seconds

- 3: Unable to extend the wrist with a weight in hand but able to extend the wrist against gravity

- 2: Partial extension without full range of motion
- 1: Flicker of muscle activation

$\circ$ 0: No movement

\section{Hand Intrinsic}

- Ask the patient to hold an empty water bottle or an empty soda can, then squeeze.

- Suggested scores are as follows:

- 5: An empty water bottle or an empty soda can is crushed

- 4: Unable to fully crush an object but able to create a dent

- 3: Able to hold an object in his or her hands without dropping the object against gravity

- 2: Unable to hold an object against gravity

- 1: Flicker of muscle activation

- 0: No movement

\section{LOWER EXTREMITY MOTOR STRENGTH EXAMINATION}

\section{Hip Flexors/lliopsoas}

- Have the patient sit in an upright (e.g., dining) chair with the knee flexed.

- Have the patient attempt to flex his or her hip to raise the thigh off the chair and hold.

- Alternatively, the patient may do this standing while balancing himself or herself with a hand on a support, such as a table or countertop.

- Suggested scores are as follows:

- 5: Hold with no perceived weakness

- 4: Hold with perceived weakness or less time than other side

- 3: Can lift but not hold

- 2: Can only flex hip without gravity (e.g., lateral decubitus)

- 1: Trace, palpable, or visible contraction

- 0: No movement

\section{Quadriceps}

- The preferred method is to have the patient single-leg rise out of a chair or onto a step/step stool, with support.

- An alternative for more frail patients for whom single-leg stance maneuvers may not be performed safely is a seated knee extension.

- Suggested scores are as follows:

- 5: Can complete maneuver with no perceived weakness

- 4: Can complete maneuver with perceived weakness and/or weaker than other side 
- 3: Able to fully extend knee while seated

- 2: Can only extend knee without gravity (e.g., lateral decubitus)

- 1: Trace/palpable or visible contraction

○ 0: No movement

\section{Hamstrings}

- While standing upright, have the patient actively flex his or her knee as far as possible, using the contralateral hand for balance on a table as necessary. If necessary, the patient may use the ipsilateral hand to help passively flex the knee, but then release the hand. The patient should maintain this position for 30 seconds or as long as possible.

- Suggested scores are as follows:

- 5: Able to hold knee flexed with no perceived weakness

- 4: Able to hold knee flexed with perceived weakness/asymmetry

- 3: Able to flex knee but not hold

- 2: Able to flex knee only without gravity (e.g., lateral decubitus)

- 1: Trace/palpable or visible contraction

- 0: No movement

\section{Ankle Dorsiflexion/Tibialis Anterior}

- Have the patient stand on his or her heels and, if able, walk several paces, using a wall or table for balance as necessary.

- Suggested scores are as follows:

- 5: Able to stand/walk on heels (with wall support) and clear metatarsal heads

- 4: Able to stand/walk on heels without clearing metatarsal heads

- 3: Able to actively dorsiflex ankle while seated

- 2: Able to actively dorsiflex ankle only without gravity (e.g., lateral decubitus)

- 1: Trace/palpable or visible contraction of tibialis anterior

○ 0: No movement

\section{Ankle Plantarflexion/Gastrocnemius/Soleus}

- Have the patient support himself or herself with an arm stretched out to a wall for balance, then stand on one leg. Ask the patient to raise the heel off of the floor as fully as possible.

- Suggested scores are as follows:
- 5: Able to stand/walk on toes with no perceived weakness (single-heel rises can be repeated up to 10 times to detect subtle weakness)

- 4: Able to stand/walk with perceived weakness/asymmetry

- 3: Unable to rise onto toes/clear heels

- 2: Able to plantar-flex foot while seated with leg raised/foot off floor

- 1: Trace/palpable or visible contraction of gastrocnemius/soleus

○ 0: No movement

\section{REFLEXES}

We have not identified a reliable method of reproducing the reflex examination, with the exception of the Babinski response, which can be assessed by having the assistant stimulate the bottom of the patient's foot and observing for fanning of the toes and extension of the great toe. Most patients with significant myelopathy have difficulty performing tandem walk (i.e., heel to toe walking). This examination can be useful in identifying patients with significant cervical or thoracic cord compression causing myelopathy. If a family member is present, a gentle tap below the knee hitting the patellar tendon can also elicit a significant knee jerk indicating possible myelopathy.

\section{TENSION SIGNS}

To elicit tension signs from the patient, an active assistant is required in order to complete this portion of the examination. If an assistant is not available, the patients can be directed to palpate over bilateral sacroiliac (SI) joint and spinous processes, and these can be documented separately.

\section{Straight Leg Raise}

- Ask the patient to lie flat, and ask the assistant to raise the leg with knee extended into air. If reproduction of shooting pain below the knee occurs in a sciatic nerve distribution beyond $30^{\circ}$ of hip flexion, the test is considered positive.

\section{Femoral Stretch}

- Ask the patient to lie on the side, and have an assistant extend the leg at the hip with knee 
flexed. If upper thigh pain is reproduced, then the test is considered positive.

\section{Spurling Sign}

- Ask the patient to extend the neck as completely as possible. Reproduction of preoperative radicular symptoms with rotation to the ipsilateral side is considered positive.

\section{Lhermitte Sign}

- Lhermitte test can be performed by having the patient flex and extend the cervical spine and assessing the patient for electrical-like truncal symptoms.

\section{Palpation Over SI Joint}

- Ask a patient to compress his or her SI joint, then report reproduction of pain.

\section{Palpation at the Cervical/Thoracic/Lumbar Spinous Processes}

- Ask a patient to palpate over spinous processes of cervical, thoracic, and lumbar spine and report reproduction of pain.

\section{Range of Motion}

- Ask a patient to perform flexion/extension/ rotation/side bending of his or her neck and report pain if elicited along with limitation of motion.

- Ask a patient to perform flexion/extension/ side bending and side-to-side bend of his or her torso and report pain if elicited along with limitation of motion.

These are only guidelines; therefore, each investigator should use his or her own clinical judgement to determine the appropriate examination exercises for their patients. Suggested scores are intended to be consistent with the scoring criteria in the Neurologic Examination. ${ }^{9}$

\section{KEY TAKEAWAYS}

Telemedicine helps to establish a working patientprovider relationship prior to the in-person visit. The telemedicine visit effectively gathers necessary information, including pertinent patient history and physical examination findings. The examining physician can then direct the patient for further studies or for an emergency visit if significant or progressive neurologic deficits are identified. The use of telemedicine for spinal examinations should reduce the number of patient examination rooms required in the outpatient setting because physicians have the ability to remotely screen patients. Furthermore, telemedicine will make postoperative visits more convenient for patients because of the economic benefits in saving time and money. Lastly, telemedicine will ease the process of recording postoperative visits, helping to meet the Center for Medicare \& Medicaid Services' global surgery code requirements.

The main drawbacks with spine telemedicine visits include the loss of direct physical examination, the potential for not detecting subtle neurologic deficits, and technical software or hardware difficulties. These technical shortfalls may be ameliorated by improving network speed, accessibility, and upgrading software usability. Synchronizing EMRs with a telemedicine application could also improve upon currently existing telemedicine platforms.

\section{DISCUSSION}

In times of national health care emergencies, the complex web of social needs, health care availability, and accessibility to technology is dynamic. For example, nonurgent and nonemergent spinal surgeries are being deferred in some geographic areas until the crisis abates. However, it is difficult to determine the degree of neurologic urgency without performing an examination. This protocol is designed to incorporate accepted neurologic and spinal examination principles via telemedicine and establish a guideline to facilitate the examination process. The reasons for difficulty in establishing a strict guideline lie in the art of history-taking and physical examination for patients with spine disease. Variations in the practice of spine care are magnified when the provider cannot talk to or examine the patient in person. Because there is no standard method to accurately, reliably, and consistently perform a spine examination through telemedicine, this paper suggests a formal spinal examination that is both user-friendly and nearly comprehensive.

The psychologic effect of the COVID-19 pandemic for patients who are in unrelenting pain or who have progressive neurologic deficits cannot be understated. For this subset of spine patients, inperson evaluation in an emergency room may be more appropriate rather than in an office setting. 
Prior to scheduling a patient for a telemedicine visit, a triage system is needed to screen patients to determine if they are deemed safe and appropriate to undergo telemedicine evaluation electively or if they need to be seen in the emergency room. At the conclusion of the visit, a spine provider should decide if a patient would need an emergent or an urgent surgery based on Elective Surgery Acuity Scale recommended by the American College of Surgeons. ${ }^{10(\mathrm{p} 19)}$ By no means can telemedicine replace all in-person visits, but it can be a valuable tool to reach most spine patients during this crisis. Data privacy is a huge concern with telemedicine software, which has garnered much interest in the media recently. ${ }^{11}$ The breach of personal health information can occur despite multiple layers of security that are built into these telemedicine portals. However, given the situation, these are the risks that both the providers and the patients must discuss and acknowledge before proceeding with telemedicine visits.

The use of telemedicine in outpatient settings across a variety of medical and surgical specialties is increasing given the need for social distancing in the era of COVID-19. ${ }^{12}$ As more physicians adopt telemedicine into their clinical practice, patients will become more comfortable interacting with their physicians via an online platform. Even after the current pandemic recedes, we expect that telemedicine will become more widely used because of its economic benefits for patients, efficiency for practitioners, and facilitation of postoperative care. By presenting a standardized remote spinal examination in this manuscript, we hope that spine care providers will be able to use this methodology to perform full spine evaluations through telemedicine.

\section{SUPPLEMENTAL METHODS}

Cervical exam: https://youtu.be/HvkLssBFzkU

Lumbar exam: https://youtu.be/tyDXVaqygKA

\section{REFERENCES}

1. Lempinen E. COVID-19: economic impact, human solutions. Berkeley News. https://news.berkeley.edu/2020/04/10/ covid-19-economic-impact-human-solutions/. Published April 11, 2020. Accessed April 23, 2020.

2. Nakajima I. Telecommunications for disasters and pandemics. New Breeze. 2013;(Spring):21.

3. Awadallah M, Janssen F, Körber B, Breuer L, Scibor M, Handschu R. Telemedicine in general neurology: interrater reliability of clinical neurological examination via audio-visual telemedicine. Eur Neurol. 2018;80(5-6):289-294.
4. Wechsler LR, Tsao JW, Levine SR, et al. Teleneurology applications. Neurology. 2013;80(7):670-676.

5. Condon A. Rothman Orthopaedics ramps up telemedicine but physical exam still challenging for spine, says Dr. Gregory Schroeder. Becker's Spine Review. https://www.beckersspine. com/orthopedic-spine-practices-improving-profits/item/48698rothman-orthopaedics-ramps-up-telemedicine-but-physical-ex am-still-challenging-for-spine-says-dr-gregory-schroeder.html. Accessed April 23, 2020.

6. COVID-19 \& HIPAA Bulletin: limited waiver of HIPAA sanctions and penalties during a nationwide public health emergency. March 2020. https://www.hhs.gov/sites/default/ files/hipaa-and-covid-19-limited-hipaa-waiver-bulletin-508.pdf. Accessed April 23, 2020.

7. Mani S, Sharma S, Singh DK. Concurrent validity and reliability of telerehabilitation-based physiotherapy assessment of cervical spine in adults with non-specific neck pain [published online ahead of print July 4, 2019]. J Telemed Telecare. doi:10. $1177 / 1357633$ X 19861802

8. Krishna C, Livingston A, Holman P, Benzel E. Chapter 12: physical and neurologic examination. In: Spine Surgery: Techniques, Complication Avoidance, and Management. Vol. 1. 3rd ed. Philadelphia, PA: Elsevier Saunders; 2012:112-115.

9. McDonald J, Welch WC. Chapter 3: patient history, general physical examination, and neurologic examination with studies. In: Operative Spine Surgery. Vol. 1. 1st ed. New York, New York, United States: Appleton and Lange; 1999:13-26.

10. American College of Surgeons. COVID-19: guidance for triage of non-emergent surgical procedures. https://www.facs. org/covid-19/clinical-guidance/triage. Accessed April 27, 2020.

11. Hodge R. Zoom security issues: Zoombombings continue, with racism and child abuse. CNET. https://www.cnet.com/ news/zoom-security-issues-zoombombings-continue-include-rac ist-language-and-child-abuse/. Accessed April 27, 2020.

12. Goz V, Spiker WR, Brodke D. Mobile messaging and smartphone apps for patient communication and engagement in spine surgery. Ann Transl Med. 2019;7(suppl 5):S163. doi:10. 21037/atm.2019.08.10

Disclosures and COI: The authors declare no conflicts of interests with this manuscript. This research received no specific grant from any funding agency in public, commercial, or not-for-profit sectors.

Corresponding Author: Jang W. Yoon, MD, MS, Perelman School of Medicine, University of Pennsylvania, Department of Neurosurgery, 3400 Civic Center Blvd., Philadelphia, PA 19104. Phone: 215-829-6700; Fax: 215-829-6645; Email: jang. yoon@pennmedicine.upenn.edu.

Published 30 June 2020

This manuscript is generously published free of charge by ISASS, the International Society for the Advancement of Spine Surgery. Copyright (C) 2020 ISASS. To see more or order reprints or permissions, see http://ijssurgery.com. 